\title{
CCF THPC inaugural issue editorial
}

\author{
Depai Qian ${ }^{1} \cdot$ Dhabaleswar K. Panda ${ }^{2}$
}

Published online: 8 May 2019

(c) China Computer Federation (CCF) 2019

High performance computing (HPC) has been one of the primary drivers for advancing modern scientific research and development. For example, using numerical simulations enabled by HPC, scientists are now able to conduct predictive research, which allows a better understanding of the essence of nature and discovering the law of the physical world. Many engineers now exploit HPC in their design and optimization of novel products and complex engineering schemes, and such simulation-based approaches are much more superior to the traditional prototyping methods. Research on grand challenges such as energy shortage, environment pollution, and climate change are usually conducted with extensive scenario simulation, data analysis, and decision-making process, which is impossible without supercomputing. Furthermore, HPC also benefits ordinary people with accurate weather forecasting, new drug development and precision medicine being an indispensable part of our daily life, which all heavily rely on HPC. Nowadays, it is hard to find a corner in our society that has not been influenced or re-shaped by HPC.

Because of its crucial importance in social and economic development, HPC has been identified as one of the priority research subjects. National R\&D programs on HPC have been proposed and implemented in China and in many other countries such as the United States, Japan, and EU countries. Great efforts including funding and manpower have been invested in this field. As a result, we have witnessed tremendous progress on HPC in the past 20 years in China and all over the world.

HPC is by no means a single discipline. Instead, it involves a number of different disciplines, for example, mathematics, computer sciences, software engineering, information security, and domain sciences. The topics related to HPC include computational methods, parallel algorithms,

\footnotetext{
Depai Qian

depeiq@buaa.edu.cn

Beihang University, Beijing 100191, China

2 Ohio State University, Columbus, OH 43210, USA
}

parallel computer architectures, processor micro-architectures, memory and storage systems, high performance interconnect, efficient power supply, system cooling, reliability, parallel programming, application software development environment, and domain application software, etc. All the above mentioned topics will be covered by this journal, the CCF Transaction on High Performance Computing (CCF THPC). With CCF THPC, we aim to establish a platform for the international HPC communities to exchange research experiences, share knowledge, and discuss important issues by publishing high-quality papers in this journal. This is also the motivation of China Computer Federation (CCF) to launch such a journal.

Currently, we are developing towards exascale computing, another milestone along the HPC journey. There are a number of grand challenges in developing the exascale computer systems, among them, the power consumption, performance obtained by applications, programmability, and reliability are the most dominant issues. We need to attack those challenges from various aspects, including developing creative architectures, novel processor, memory and interconnect technologies, new devices, architecture-aware algorithm implementation, large-scale parallel programming, performance tools, and computable modeling of the target systems, etc. And also, disruptive architecture and technologies are expected, which might change the landscape of HPC in the coming years. This is truly a multi-disciplinary and international joint effort. We encourage international scholars working on HPC to submit the original papers about their research and development to this journal. The quality of the journal truly relies on the enthusiasm and excellence of the authors.

The CCF THPC editorial board team and the editorial workflow are organized as follows. The journal follows a two Editor-in-Chief (EiCs) approach, one from China and the other from the United States. Two Managing Editors are appointed to assist the EiCs on the regular management tasks. The advisory board consists of a group of senior and distinguished scholars, who will provide advice and guidance to the journal. The editorial board team is formed with 
30 high-profile researchers in the concerned fields and covers the major HPC research labs from more than nine countries. The advisory board and the members of the editorial board, who handles the reviews of the regular papers are listed on the inside cover of this issue. The Guest Editors, mostly invited specialists, are involved in selecting interesting topics for special issues and handle reviews of the themeoriented papers. These absolutely first-rate Guest Editors not only make recommendations on the acceptance or rejection of papers, but also help improve the quality of the papers by providing a constructive and timely review process.

This inaugural issue focuses on the topic of Exascale computing. Three state report papers from China, the United States, and the European Union introduce the national R\&D programs on exascale computing in the respective countries. Authors can find useful information about the newest activities and progress of those ambitious programs. The report from another important player in Exascale computing, that is, Japan, will appear in the later issue. One paper on parallel programming framework reflects the effort of Chinese researchers on reducing difficulty in programming the exascale computers and improving the productivity of developing large-scale parallel application software. One paper from the Gordon Bell Prize 2017 winner gives the design and implementation strategy of their wining applications on earthquake simulation over Sunway Taihulight. One paper presents a class of space-time domain decomposition methods for solving parabolic problems at the extreme-scale. Another paper studies I/O issues of data-intensive scientific applications in exascale computing. The remaining three issues in the 2019 year volume will touch other key HPC topics, including advanced HPC technologies and applications, AI and HPC, and storage systems to be published in June, September, and December, respectively.

We look forward to seeing CCF THPC establish itself as the premier venue for publishing original research, stateof-the-art cross-disciplinary surveys, and reviews on the emerging areas of HPC technologies and applications. Ultimately, the success of this journal depends on the effort of international HPC communities and the individual editors and authors, who subscribe, submit, review, read, and cite the papers. We appreciate your contributions and very much look forward to your continuous support!

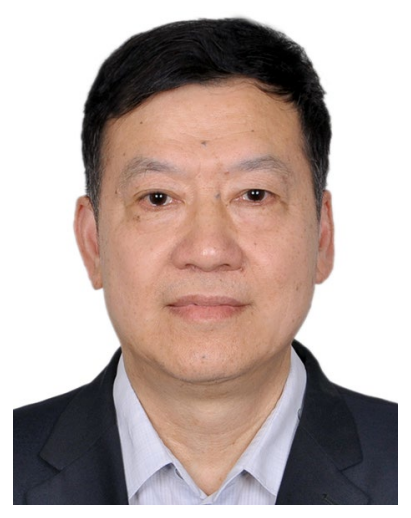

Depai Qian professor at Sun Yatsen University and Beihang University, dean of the School of Data and Computer Science of Sun Yat-sen University. Since 1996 he has been serving as the expert of the national $R \& D$ program of China and is currently the chief scientist of the national key project on high performance computing. He has been working on computer architecture and computer networks for many years. His current research interests include high performance computer architecture and implementation technology, distributed computing, and performance measurement and evaluation. He has published more than 300 papers in journals and conferences.

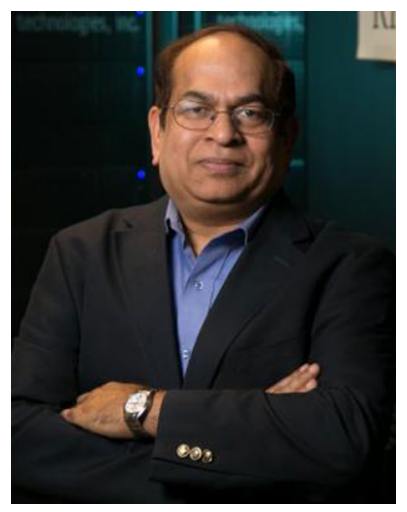

Dhabaleswar K Panda is a Professor and University Distinguished Scholar of Computer Science and Engineering at the Ohio State University. He has published over 450 papers in the area of high-end computing and networking. The MVAPICH2 (High Performance MPI and PGAS over InfiniBand, OmniPath, iWARP and RoCE) libraries, designed and developed by his research group (http://mvapi ch.cse.ohio-state.edu), are currently being used by more than 3,000 organizations worldwide (in 86 countries). More than 538,000 downloads of this software have taken place from the project's site. This software is empowering several InfiniBand clusters (including the 3rd, 14th, 17th, and 27th ranked ones) in the TOP500 list. The RDMA packages for Apache Spark, Apache Hadoop and Memcached together with OSU HiBD benchmarks from his group (http://hibd.cse.ohio-state.edu) are also publicly available. These libraries are currently being used by more than 305 organizations in 34 countries. More than 29,900 downloads of these libraries have taken place. High-performance and scalable versions of the Caffe and TensorFlow frameworks are available from https://hidl.cse.ohiostate.edu. Prof. Panda is an IEEE Fellow. More details about Prof. Panda are available at http://www.cse.ohio-state.edu/ panda. 